Article

\title{
Analysis of Modal Parameters Using a Statistical Approach for Condition Monitoring of the Wind Turbine Blade
}

\author{
Lukasz Dolinski *,+(D) and Marek Krawczuk + \\ Department of Mechatronics and High Voltage Engineering, Faculty of Electrical and Control Engineering, \\ Gdansk University of Technology, 80-233 Gdansk, Poland; marek.krawczuk@pg.edu.pl \\ * Correspondence: lukasz.dolinski@pg.edu.pl \\ t These authors contributed equally to this work.
}

Received: 15 July 2020; Accepted: 22 August 2020; Published: 25 August 2020

\begin{abstract}
The primary objective of the presented paper is the numerical and experimental investigation related to developing a useful diagnostic method, which can be used for determining the site and size of damage in laminated shells of wind turbine blades. The described detection technique is based on the analysis of low frequencies bending vibrations mode shapes of rotor blades. The authors used the commonly applied statistics methods that have been adapted to detect edges of damage, including the normalized determination coefficient fit, which is a measure of the absolute fit between two curves. The research was conducted for a scaled-down blade of a three-bladed horizontal-axis wind turbine with $36 \mathrm{~m}$ diameter rotor. The study was divided into two parts. The first stage included numerical calculations using the finite element method, which were supplemented in the second stage by measurements under laboratory conditions of the specially manufactured composite blade. The forms of natural vibrations for intact and damaged blade were determined using Laser Doppler Scanning Vibrometry. The results of the presented research confirm the effectiveness of the modal analysis combined with statistic calculation in damage detection. The method points out the location of relatively small damage.
\end{abstract}

Keywords: modal analysis; non-destructive damage detection; wind turbines blade; vibration based methods; statistic; laser vibrometry

\section{Introduction}

Nowadays, there is intense social pressure to decrease the negative influence of human civilization on the natural ecosystem, especially in terms of electricity production. The use of renewable energy sources is deemed a necessity. On the other hand, with increasing environmental requirements and, at the same time, growing demand for electricity, there are also increasing requirements on the security of energy supply and its quality. Therefore, despite some drawbacks, wind energy is still one of the essential elements of the electricity production system using clean power. Unfortunately, wind turbines (WTs) have significant weaknesses compared to non-renewable energy resources. The primary issue is the lower efficiency due to limited working time at variable and unpredictable weather conditions. An attempt to eliminate those drawbacks by increasing the rotor diameter or by locating wind farms in open waters introduces some factors which increase the risk of damage to critical components of the turbine mechanical system. The damage risk applies, in particular, to the blades, which are susceptible elements of the wind turbine structure, and at the same time crucial from the operating point of view. Appropriate design and manufacturing of blades makes it that the manufacturer guarantees the fatigue life for 20 to 30 years of exploitation [1-3]. However, it is not possible to predict 
all situations that could lead to damage. That is why the newly built installations are equipped with a comprehensive system for monitoring the technical condition of all of the most critical elements of the system, including the blade. The use of such a diagnostic system allows reducing the effects of possible failure, extending the life cycle of the device and even predicting the rate and direction of damage spread, which makes it possible to decide on the possibility of further operation or the need to replace the element. A large group of operating wind turbines are devices built several years ago, which are approaching the end of their life cycle and have no dedicated damage detection systems. In this case, periodic inspection is the only possibility to assess their condition. It should be pointed out that standard methods of damage detection and location in laminates such as ultrasound are time-consuming and require stopping the turbine. A large number of current publications focused on damage detecting methods for turbine blades indicates the high practical importance of this topic. A broad overview of the methods and monitoring systems used in the diagnostics of wind turbines including blades can be found in [4-6]. Issues related to the development of new diagnostic methods of wind turbine blades should be aimed at three different aspects connected with the selection of physical parameters to analysis, measurement methods and signal processing methods. A large group of researchers in the subject focuses on techniques related to the analysis of mechanical parameters such as vibroacoustic $[7,8]$, shape curvature $[9,10]$, including these which measurement is possible without contact at a long distance and no sophisticated equipment (e.g., image correlation [11] or thermography [12]). The methods of signal analysis are both a classical [13-15], as well as a novel approach using AI algorithms, etc. $[16,17]$. Many of these methods are only applicable in laboratory conditions due to the high accuracy of the measurement, time-consumption of the measurement and calculation or complex measurement equipment required [18].

The results presented by authors in this paper are part of a broader investigation on the development of a fast and effective non-destructive testing method aimed to detect, localize and identify of failures of a wind turbine rotor blade. In the first stage of general research on the diagnostic method, the one-dimensional Continuous Wavelet Transform (abbreviated CWT) was used to analyze bending forms of natural vibrations of the turbine blade. The results of this investigation can be found in [19]. The key finding of this study was that the detection method is useful only under particular conditions, mainly related to the high accuracy of the measured signal. The localization of the blade shell imperfections is only possible using a very low noise level in the signal. The second requirement concerns the full knowledge about the design of the object under investigation. However, even in the case of low noise level data obtained as a result of numerical simulation, it is needed to use signal pre-processing. The complexity of the method is also influenced by the need to analyze modes independently with individually selected parameters of signal pre-processing and CWT analysis. As a result, this method, despite its clear advantages, is complicated and time-consuming. However, knowing the approximate location of the damage, it is possible to determine its edges with reasonable precision. Therefore, as a supplement to that research, a method for preliminary damage location was proposed based on a complex analysis of the natural vibration mode shape by Root Mean Square (abbreviated RMS) calculation [20]. Despite the undoubted advantages of the method used, such as insensitivity to measuring noise or calculation speed, the interpretation of the result was not always unambiguous and clear. Nevertheless, statistical methods seem to be useful in analyzing the form of vibrations, assuming knowledge about the undamaged state of the tested object. Of course, the use of statistical tools in the analysis of vibroacoustic signals is nothing new. However, this type of research mainly focuses on the analysis of signals located in the time domain (e.g., acoustic emissions [21] or vibrations [22]) or large data sets [23]. Therefore, in the present research focused on the improvement of the diagnostic method based on modal parameters, the authors proposed to use a statistical tool such as fit coefficient. Using these statistic calculations allowed to improve the preliminary detection method for easier interpretation of the result and in some cases, even approximated determination of the damaged edge. The results of this investigation are presented in this article. 


\section{Methodology}

\subsection{General Assumptions—Research Object}

In this paper, the authors present the evolution of the previous technique for determining the location of damage in a composite wind turbine blade coating (which was described in $[19,20]$ ) using the statistic approach. The authors have already explained several aspects of the numerical and experimental research methodology. However, these aspects are still included in this section to ensure completeness. From the beginning of the works on the method of assessing the technical condition of composite coatings of wind turbine blades, the authors are guided by the assumption of use classical mechanical quantities, such as modal parameters. In particular, those, which are possible to experimentally obtain without the disassembly of the WT blade. At the same time, by using high-accuracy equipment for non-contact vibration measurements, it is possible to use a computational method that until now has not worked because of its sensitivity to noise. In addition to detecting damage, the method should also indicate the position of its appearance. For this reason, it is necessary to use signals containing information about changes at the local level, at least in one dimension of the spatial domain. Such quantities are undoubtedly mode shapes of natural vibrations of the blade. The general advantage of the damage detection method based on mode shapes analysis is independence from the technique of obtaining modal data. The modern techniques of vibration measurement allow determining the shape of the form with an adequate precision with a measurement time acceptable from the practical point of view. Vibration measurements can be performed using laser vibrometry supplemented by additional devices, such as is a derotator equipped with a special optical system, which synchronizes the laser beam with the rotation of the object under investigation. In recent years, the motion amplification technology, which can also be the basis of the presented method, has been very actively developed. The vibrations modal parameters can also be obtained from an operating wind turbine based on data collected from a system of piezoelectric or fibre optic sensors. Such measurement systems very often are an integral part of the modern blade structure. The basic motions of wind turbine blades can be divided into three types of vibrations depending on their orientations relative to the rotor plane. The following can be distinguished: bending in the plane perpendicular to the rotor plane, bending in the rotor plane and torsion. All three types of movements are schematically shown in Figure 1.

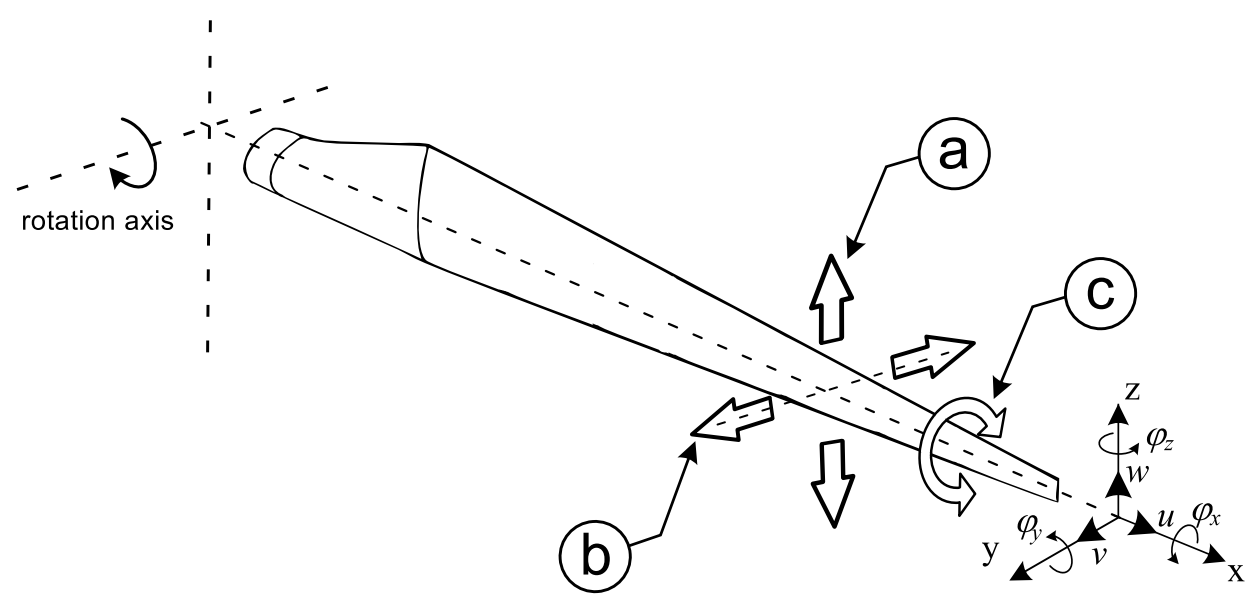

Figure 1. General motion of the wind turbine blade: $\mathbf{a}-$ bending in the plane perpendicular to the rotor plane, $\mathbf{b}$-bending in the rotor plane, $\mathbf{c}$ - torsion of the blade.

Due to the blade's profile position relative to the rotor plane, the most significant deformations of the blade exist with vibrations in the direction of the axis of rotation. Therefore, this direction of vibrations was chosen for spatial signal analysis. In the present study, the authors did not include other types of vibrations. As noted, most researchers dealing with similar topics focus on the analysis 
of particular forms of vibration. However, the conclusions of previous authors' research, as well as literature sources, suggest there is a significant correlation between the impact of damage on the shape of vibrational forms and the ordering of modes. The best results of the detection process were obtained only when the damage site and local signal extremes overlap. The solution of this problem seems to be analysing the modes with more complex shapes (more local extremes), and in this way increasing the probability that the damage will be at the point of maximum deformation of the blade shape. However, these complex modes forms occur at higher frequencies, which is associated with a decrease in vibration amplitude. The second approach, which was introduced in the paper, is a more complex simultaneous statistical analysis of the first few forms of blade vibrations. A general research methodology scheme is illustrated in Figure 2.

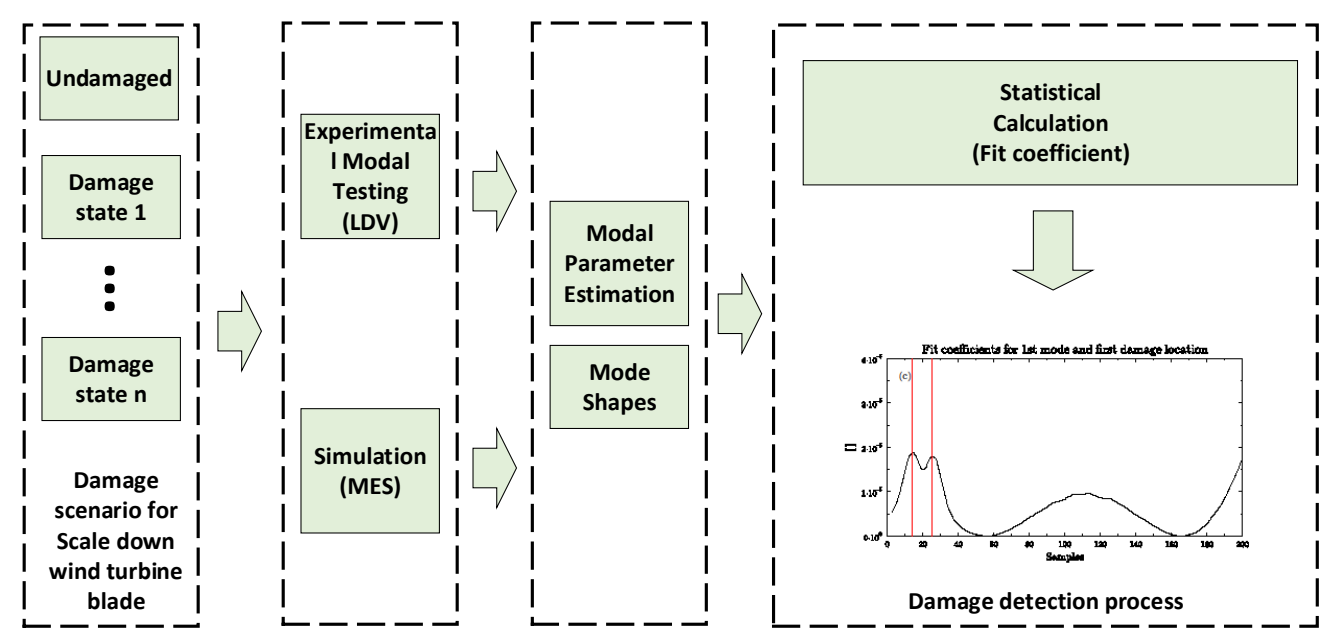

Figure 2. Research methodology.

The scope of the conducted research included both determinations of the natural vibration form by numerical simulations, which were conducted using applications of ANSYS software [24], as well as experiments with the use of Laser Scanning Doppler Vibrometer. In total, five scenarios of damage location of the blade coating for simulation calculation and three scenarios of damage for an experimental part of the research were taken into account. The undamaged state was also registered. Details of the individual stages of the study are presented further in the paper. The collected data were analyzed using statistical tools, examining the rate of their cross-fitting. All calculations were conducted using applications of MATLAB ${ }^{\circledR}$ software [25].

\subsection{Fit Coefficient}

The curves representing a particular order of vibration shape form (for a damaged and an intact blade) or the set of modes shapes obtained thanks to the numerical simulation, as well as those obtained experimentally, had been qualitatively assessed. The authors employed statistical tools that are commonly used by many researchers in signal processing. For that purpose, for each combination of two curves, a coefficients fit ware calculated, that contains information about their cross-similarity. Normalized determination coefficient $f$ it is a measure of the goodness of fit between test and reference data. The coefficient $f$ it is very often used in technical problems. The value of this coefficient is defined as:

$$
f_{i t} \text { coef }=\frac{\left\|c_{1}+c_{2}\right\|^{2}}{N}
$$

where $c_{1}$ and $c_{2}$ denotes of examined curves, $N$ denotes of number of samples and \|\| indicates the 2-norm of a vector. 
In most cases, the calculation of the fit coefficient involves comparing the two signals in the full range of available samples, thus obtaining a global similarity index for the whole signal. To obtain information on the local difference (or similarity) between the damaged signal and the reference signal (which was an important assumption of this research) the fit coefficient was calculated as a function of length. For this purpose, the fit value was determined by the one-dimensional sliding window algorithm with defined frame size. In these cases, components of the Equation (1) are noted as:

$$
f i t_{c o e f}(x)=\frac{\left\|c_{1}\left(x_{k}, x_{k+1}, \ldots, x_{k+W_{s}}\right)+c_{2}\left(x_{k}, x_{k+1}, \ldots, x_{k+W_{s}}\right)\right\|^{2}}{W_{s}}
$$

where: $c_{1}\left(x_{k}\right)$ and $c_{2}\left(x_{k}\right)$ denotes of vibration amplitude of examined states for a sample number $k=\left(1,2, \ldots, N-W_{s}\right)$ and $W_{s}$ denotes of sliding window size.

\subsection{Standardization}

During the investigation, it was observed that one of the crucial elements of the process of analyzing the modes shape of vibrations by the above-presented methods is the normalization process of signal amplitude. The need to carry out initial signal processing is because the amplitude of the natural vibrations decreases with increasing frequency. The normalization procedure equalizes the influence of all shapes modes on the statistic calculations. Following a series of calculations, it was determined that the most reliable results of damage location are obtained with the $z$-score standardization method. According to the definition, the z-scores is a measure of the distance of a particular sample from the mean for the whole signal in terms of the standard deviation and is defined as:

$$
Z(x)=\frac{c\left(x_{k}\right)-\bar{c}}{S}
$$

where: $c\left(x_{k}\right)$ denotes of vibration amplitude for a sample $k=(1,2, \ldots, N)$ of the examined mode, $\bar{c}$ denotes mean value of mode and $S$ denotes of standard deviation.

\subsection{Averaging}

Basically, the examination of each mode shape of vibration separately is time-consuming and inefficient. The solution to this issue could be the analysis of all the available mods at once. It can be achieved by calculating the RMS for each measuring point from all modes. RMS is widely used as a basic statistical tool to calculate the signal error, standard deviation or average power of the signal. Application of RMS in the analysis of modal parameters makes it possible to include information from all forms of the vibration in one single data set. Registered displacement of the particular sample for all modes was used to calculate the RMS value as:

$$
R M S=\sqrt{\sum_{i=1}^{M} \frac{c\left(x_{k}\right)^{2}}{M}}
$$

where: $c\left(x_{k}\right)^{2}$ is a squared vibration amplitude for a sample (point) $k$, and $M$ is a number of modes for RMS calculation.

\section{Results and Discussion}

In the present section, the proposed method was used to detect and locate the damage of the wind turbine blade coat. As an object under investigation, the blade of a reduced scale (1:10) of real wind turbine rotor with $36 \mathrm{~m}$ diameter was used. The blade has 1.74-m length, with one longitudinal spar and without the twist of profile. The blade design is based on the classic ClarkY aerodynamic profile. The current investigation concentrates on the impact of the defects located in part, which is responsible for the generation of the lifting force and is the most exposed to the mechanical and fatigue damage. 
The aerodynamically active part of the blade is measured from the broadest chord line of the profile to the tip (blade nose). The length of the midspan examined object was $1.44 \mathrm{~m}$. The common data for both stages of the study are presented below. Figure 3 shows the general scheme of the tested object.

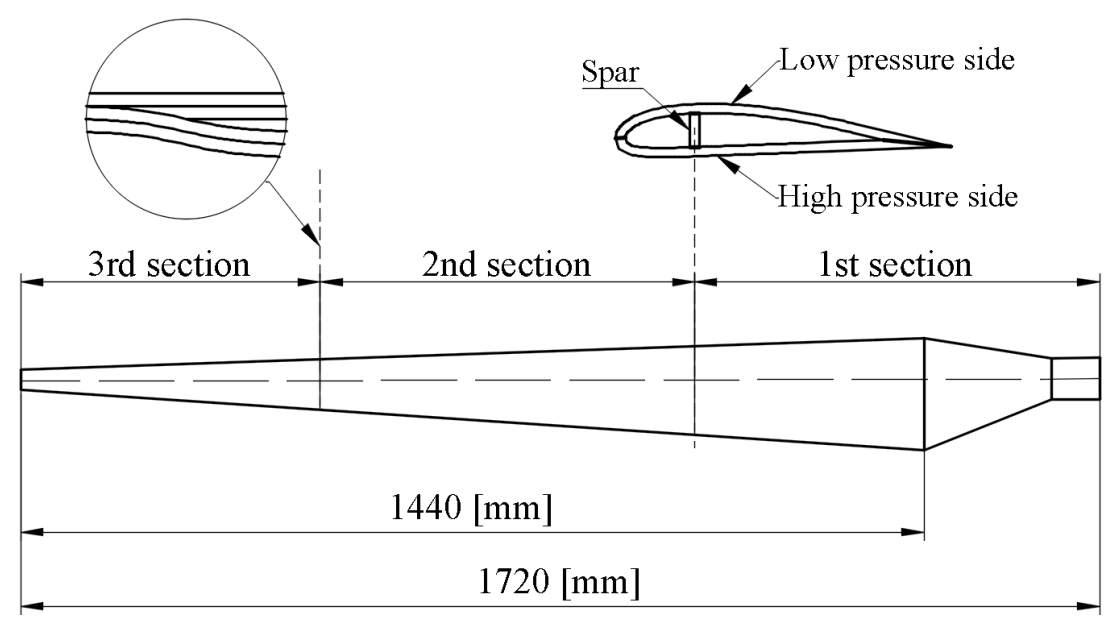

Figure 3. Scheme of the research object.

The tested blade was built in the form of a laminate shell, of which components were the fibreglass and epoxy resin. The reinforcing fibres were symmetrically arranged as $\left[ \pm 45^{\circ}\right]_{s}$. Based on the design assumptions for full-size blades, both in the numerical model and the object used in the experiment, the variable thickness of the blade coating was adopted [3,26]. The blade was divided lengthwise into three parts characterized by different thickness of the shell. Decreasing thickness was achieved by varying the number of laminate layers while allowing for a smooth thickness transition between adjacent sections. The total mass of the blade was about $2 \mathrm{~kg}$. Tables 1 and 2 show geometrical features of the tested blade as well as mechanical properties of the used composite, respectively.

Table 1. Geometrical features of the blade.

\begin{tabular}{cc}
\hline & Dimension [mm] \\
\hline Total length & 1740 \\
Max width & 160 \\
Tip width & 32 \\
Measured length & 1440 \\
Thickness of spar & 2.0 \\
Thickness of Section 1 (near blade root) & 2.0 \\
Thickness of Section 2 (middle part of blade) & 1.5 \\
Thickness of Section 3 (near blade tip) & 1.0 \\
\hline
\end{tabular}

Table 2. Properties of composite materials components.

\begin{tabular}{ccc}
\hline & Epoxy Resin & Glass Fiber \\
\hline Young modulus, E & $3.43 \mathrm{GPa}$ & $66.5 \mathrm{GPa}$ \\
Poisson ration, $v$ & 0.35 & 0.23 \\
Kirchoff modulus, $\mathrm{G}$ & $1.27 \mathrm{GPa}$ & $27 \mathrm{GPa}$ \\
density, $\rho$ & $1250 \mathrm{~kg} / \mathrm{m}^{3}$ & $2250 \mathrm{~kg} / \mathrm{m}^{3}$ \\
\hline
\end{tabular}

\subsection{Case I: Numerical Simulation}

\subsubsection{Modelling of the Blade Using Finite Element Method}

The numerical simulation was carried out using the Finite Element Method procedure (FEM). In the FEM calculations of the rotor blade under investigation, the primary numerical model consisted 
of 5409 isoparametric shell elements. Each finite element had eight nodes and six degrees of freedom at each node. It was assumed that the boundary conditions at the blade fixing were like a cantilever beam. As the results of the numerical modelling process, the values of the first 10 bending natural frequencies and corresponding to them modes shapes of vibrations were determined for damage and undamaged states. The block Lanczos method has been used to calculate the modal parameters. A series of simulations were conducted. No artificial noise was introduced at this stage of research. The data were obtained at 200 nodal points, located on a straight line along the blade length. The blade model with the damage pattern is presented in Figure 4.

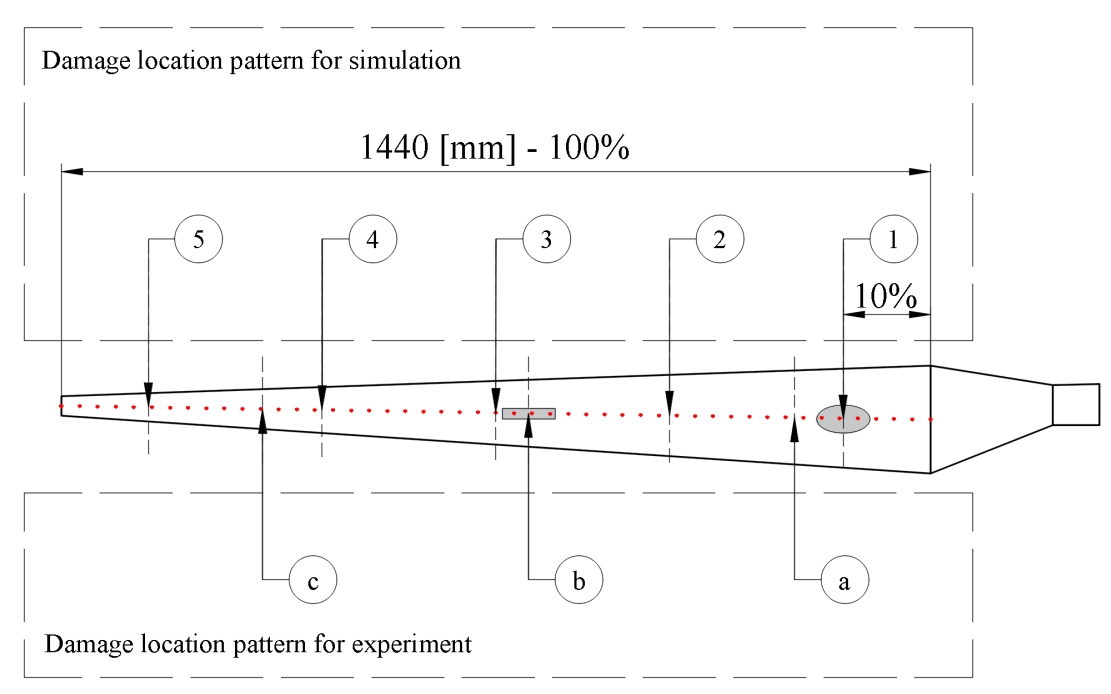

Figure 4. Damage pattern: (1-5)—numerical simulation; (a-c)—experiment.

The presented investigation concentrated only on the method for the detection of a delamination phenomenon. It is very characteristic and, at the same time, one of the most frequent types of damage for composite, especially laminated shells. The delamination means the separation of adjacent layers of composite and can be caused as a result of blade coating deformation during its motion. For this numerical study, a simple model of delamination was proposed. The concept used by the authors is associated with the phenomenon of zero tangential stress between separated layers in the damage zone. In the numerical model, this feature is simulated by reducing the shear modulus in the lamination plane $\left(G_{X Y}\right)$. The major problem in simulation process was determining the value of the shear modulus for a delaminated zone, which would provide an exact representation of the damage in the numerical model in the calculation of modal parameters with simultaneous easy implementation. For this purpose, an additional modal analysis of a straight cantilever beam with modelled damage was carried out. The obtained results were compared with the natural frequencies of the reference model. The assumed consistent of results was obtained when the shear modulus in the damaged area was lowered to $14 \%$ of its initial value. A more detailed description of the damage modeling method used can be found in [19].

\subsubsection{Damage Localization-Results}

The proposed examination procedure of vibration forms using statistic calculation consisted in determining a measure of similarity ratio between two given curves (damaged and intact). In the first stage of the investigation, the fit factors for all pairs of modes shapes were determined. Figure 5 presents results of these calculations for the blade with damage located in position 1 ( $10 \%$ of midspan measured from widest chord line of the profile) with the edges marked by vertical dashed lines. 

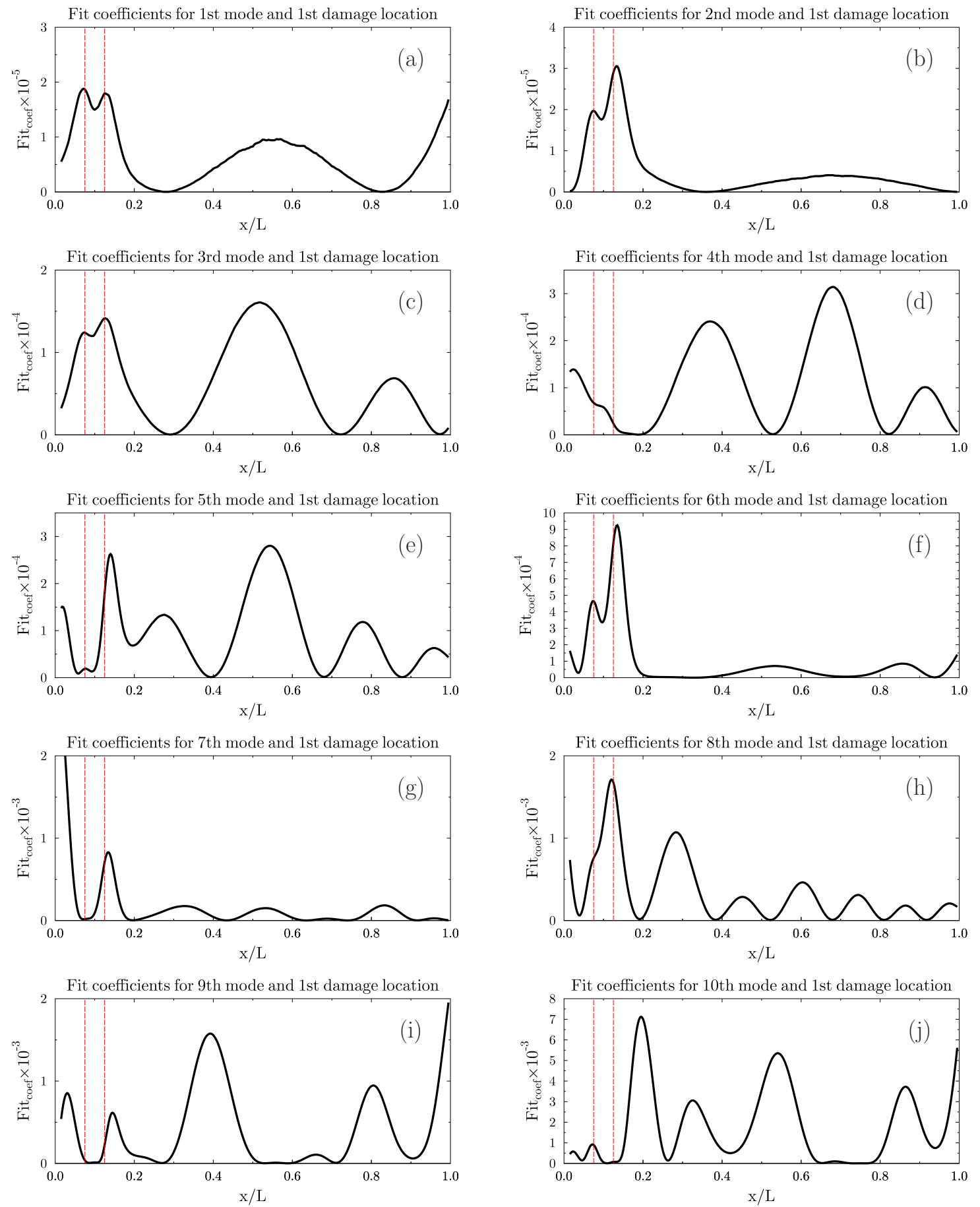

Figure 5. Results of fit coefficient calculation as a function of normalized position $(x / L)$ for modes order from 1 to 10 and the first case of damage localization: (a-j) consecutive modes; vertical dashed line-location of the damage; L-length of the measuring line.

The length of the simulated damage was $6 \%$ of the total measurement line (12 samples). The data was normalized according to Equation (3). The size of the frame for determining the sliding window method was equal to four samples, which corresponds to $2 \%$ of the total number of samples. The optimal value of the window width was determined based on a series of calculations. Analysing the results of the above calculations, the thesis that various type of modes shapes, to a different extent, contain information about the damage, is confirmed. In this case, 1st, 2nd and 6th form of vibrations include information that clearly allows for interpretation and directly indicates the limits of damage. 
In the case of the other modes, the understanding of the graph is more complicated and showing the place of damage is very difficult or even impossible. A similar relation can be seen for calculation of fit coefficient at other four locations of damage. Some examples of calculation results are presented in Figure 6.
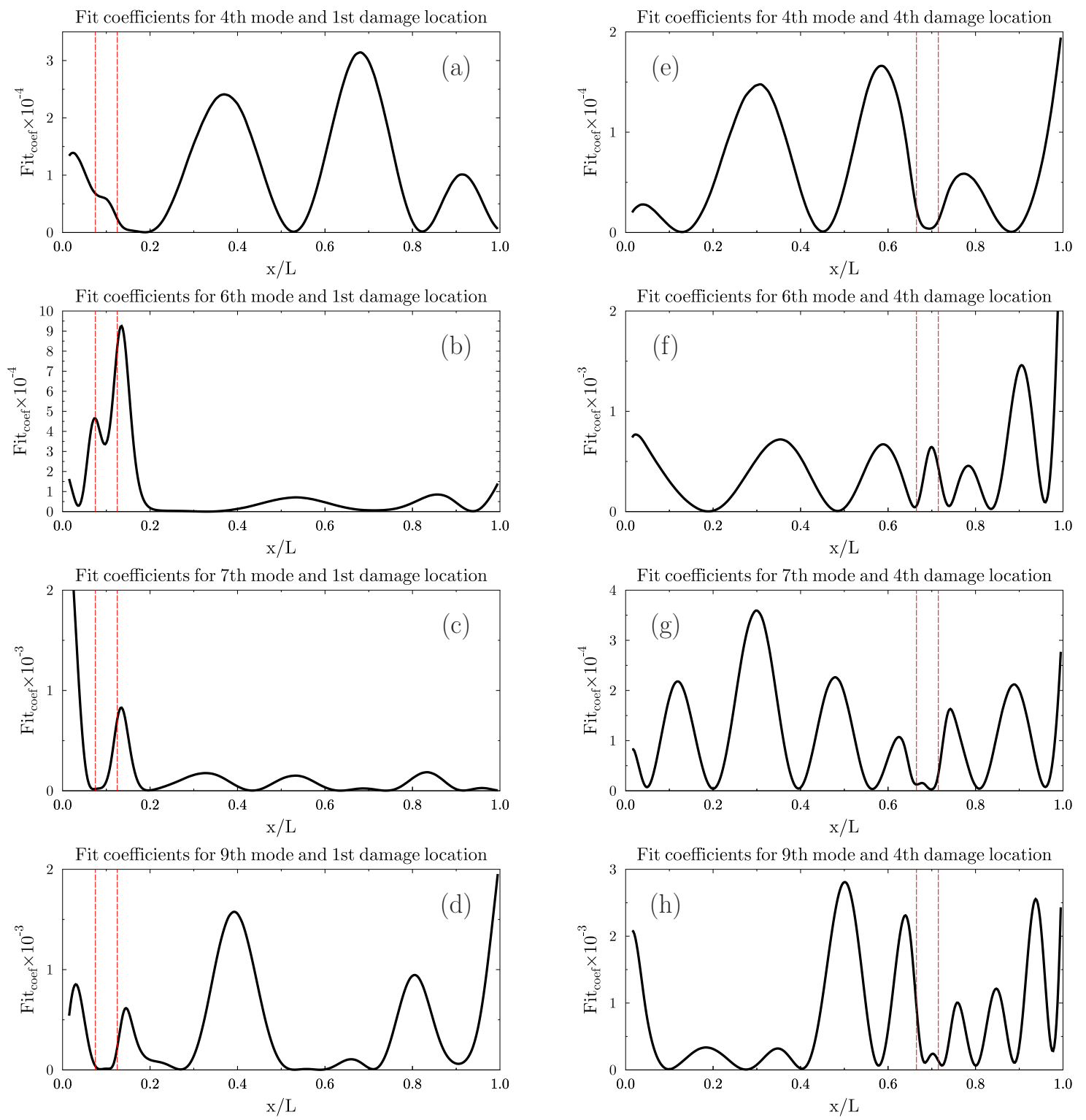

Figure 6. Results comparison of fit coefficient or individual modes (4th, 6th, 7th and 9th) for different cases of damage localization: (a-d) 1st location; (e-h) 4th location; vertical dashed line-location of the damage, $\mathrm{x} / \mathrm{L}$-normalized position; $\mathrm{L}$-length of the measuring line.

In the next step of the simulation study, the fit ratio was determined for all available mods using RMS averaging. The calculation results for the first case of delamination position (Figure 5) are shown in Figure 7. In the case of the first and the second damage location, the results were inconclusive. This effect was related to the relatively small impact of damage for total stiffness value of the cross-section near the blade root, where stiffness was high because of the widest chord line of the blade profile and the thickest coating, simultaneously. The visibility of the damage zone width on the graph increased with shifting damage position towards the tip of the blade. It is because the stiffness of the blade decreased in the blade tip direction and therefore, the relative effect of the delamination on the cross-section was more significant. 

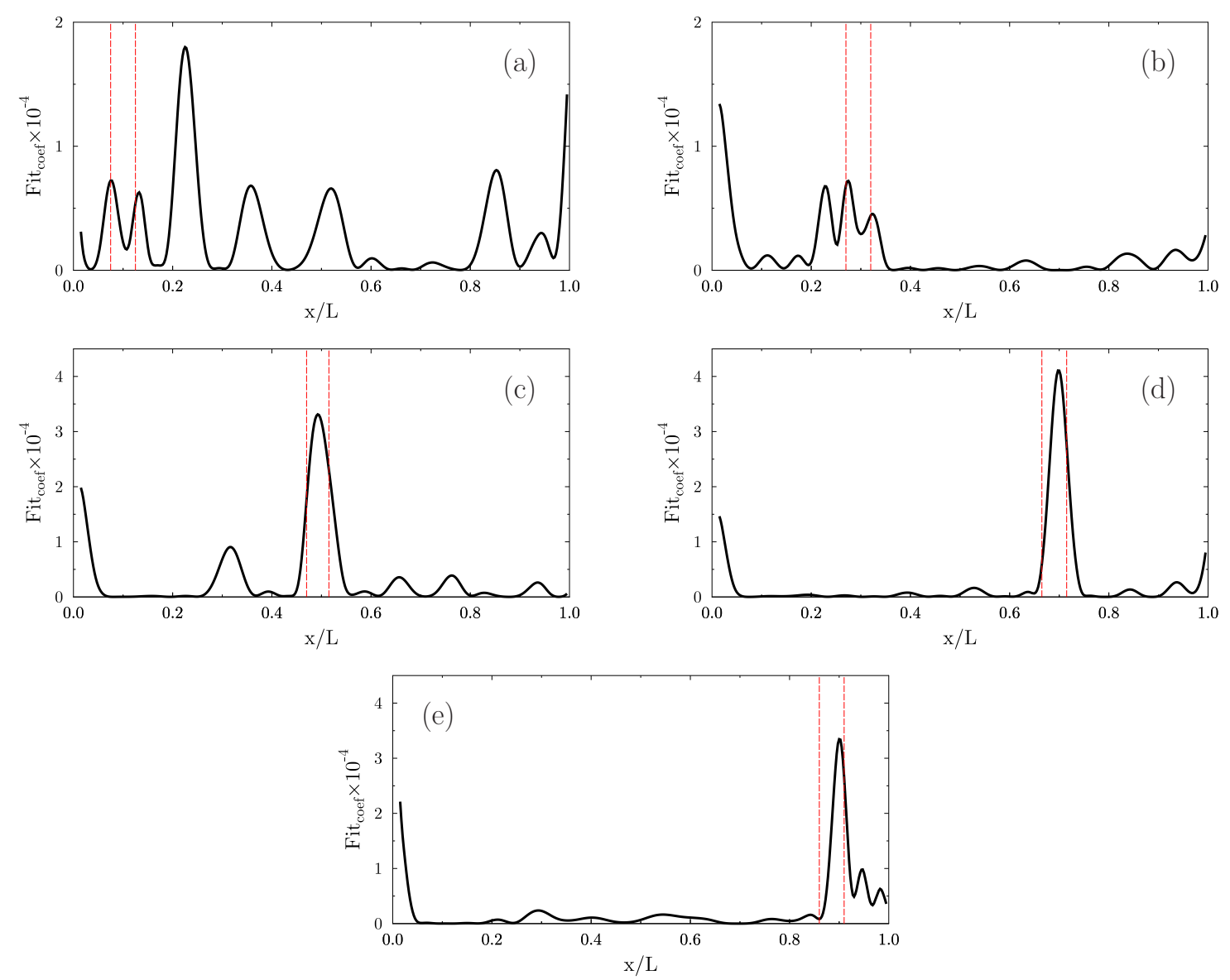

Figure 7. Results of fit coefficient calculation for averaged modes for all cases of damage localization: (a) 1st location; (b) 2nd location; (c) 3rd location; (d) 4th location; (e) 5th location; dashed line-location of the damage; $\mathrm{x} / \mathrm{L}$-normalized position.

The results of the calculation for three values of the modelled damage zone in the same location (middle of the midspan) are shown in Figure 8.

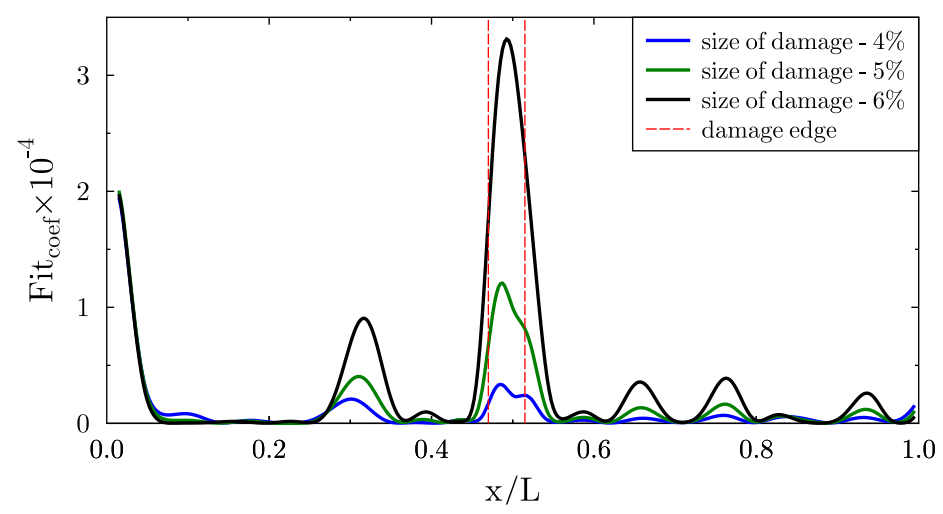

Figure 8. Results of fit coefficient calculation for RMS value for all given modes shapes and three size of damage in one localization; $\mathrm{x} / \mathrm{L}-$ normalized position.

The length of the delamination was $4 \%, 5 \%$ and $6 \%$ of the total measurement line, respectively. It can be seen that as the size of the damage decreased, the maximum value of the characteristic peak at the location of the defect also decreased. This gave the possibility not only to detect the damage but also to estimate its size. 


\subsection{Case II: Experimental Work}

\subsubsection{Experimental Setup}

As part of the presented study, the experimental measurement of the wind turbine blade in laboratory conditions was also conducted. The main purpose was verifying the results of the numerical simulation, as well as testing the damage detection method proposed by the authors on the real object. The measured blade was manufactured using a custom lay-up moulding process, with glass fibres reinforced epoxy resin as the material of the laminate skin. Figure 9 shows the scheme of the laboratory stand, which was used to measure the modal parameter of the blade. The laboratory stand included the tested object (1), an electromechanical shaker (2) and a scanning laser vibrometer with equipment (3-7).

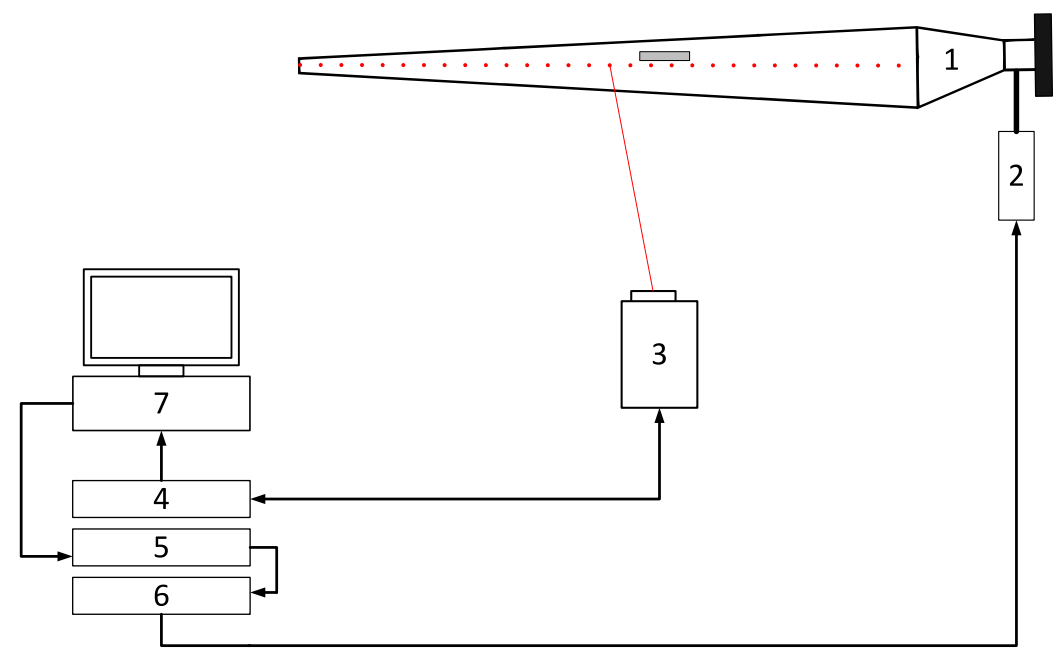

Figure 9. Scheme of the laboratory stand: (1) — tested blade, (2)—shaker, (3) - head of laser scanning vibrometer, (4)—acquisition unit (5)—signal generator, (6)—signal amplifier, (7)—computer.

The experimental identification process of modal parameters of the blade was carried out using a PSV-400 Laser Scanning Vibrometer by Polytec Ltd. [27]. The primary advantage of using this type of instrument is being fast, highly accurate and taking non-contact vibration measurements. The pictures of the measurement stand components are shown in Figure 10a-c. The most important element of the vibrometer set was the scanning head with a precise optical sensor (Figure 10b), which measured changes in the frequency of light reflected from the moving surface. Based on the principle of the Doppler effect, the vibrometer calculated velocities of vibrating objects. The significant feature of this type vibrometer was the possibility for the user to predefine the scanning grid. During the measurement, the unit automatically moved a laser beam from point to point on the surface of vibrating objects, enabling the full-field vibration analysis. Further to the scanning head, the vibrometer set also included a signal generator, a junction box and a control unit. During the tests, the appropriate measurement conditions were provided to obtain experimental data with the lowest possible noise level. The problem of the measurement noise is crucial in terms of the interpretation of the experimental data in the damage detection process. The high level of noise values can mask information about the damage and consequently can prevent its detection. Therefore, the test object was isolated from any external vibration sources by fixing it to a special table. At the same time the maximum level of the signal received by the vibrometer was achieved by wrapping the tested blade surface with a special retro-reflective foil. 


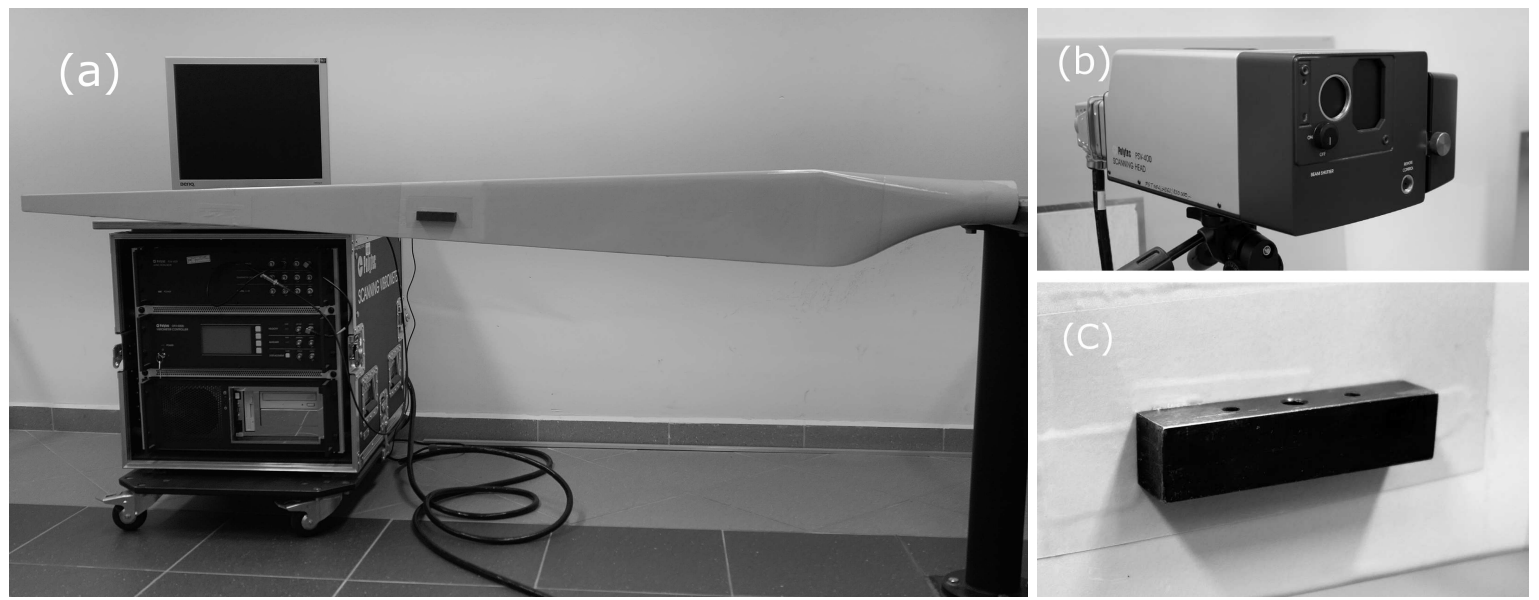

Figure 10. The experimental set up in laboratory: (a) — tested blade and equipment of LSV, (b) —head of laser scanning vibrometer, (c) - model of the damage.

The experimental part of the study included measuring the intact state of the object and three scenarios of damage location of the blade coating. A delamination zone was simulated using high stiffness steel element fixed to the blade skin on the measured side, as shown in Figure 10c. This element changed the local stiffness of the composite coating. The blade damage pattern for the experiment is presented in Figure $4-\mathrm{a}, \mathrm{b}$ and $\mathrm{c}$ locations. The first scenario relates to damage location, which is nearest to the blade fixing (Figure 4-element a). In order to minimize the possible impact of the edge effect, that could arise in the event of overlapping damage and the beginning of the analyzed signal, the position of the damage was shifted towards the centre of the blade. The same procedure was used for damage located on the opposite end of the midspan (Figure 4 element c). The last damage position was determined halfway between the extreme locations (Figure 4 element b). For all scenarios, the basic length of the damage was $6 \%$ of the total length measurement line, corresponding to 12 measurement points. Additionally, for the damage located in the blade centre, the data were supplemented with measurements for damage spanning over $5 \%$ and $4 \%$ of the total length measurement line (10 and 8 samples, respectively). The 200 measuring points were aligned to one line in the middle of the blade width, located directly under the simulated damage.

The electromechanical shaker with maximum excitation forces equal to $31 \mathrm{~N}$ was used to induced vibrations of the blade during the measurement. For this purpose, the sinusoidal signal of constant amplitude and a linearly varying instantaneous frequency range from $0 \mathrm{~Hz}$ to $550 \mathrm{~Hz}$ was generated. The first stage of the experiment procedure, which was repeated for each damage scenario, was measuring of the Frequency Response Function (FRF) with a resolution of 6400 FFT lines. The FRF function was used to determine the values of resonant frequencies. In the second step, the modes shapes corresponding to the resonant vibrations were determined separately using a sinusoidal excitation at a constant frequency and a narrow frequency bandwidth of $0.02 \mathrm{~Hz}$. The test procedure also included multiple measurements at each point of the grid (from 3 to 5) and averaging the result. This type of measurement was used to minimize the noise level and obtain high accuracy data. However, such a procedure is associated with an extended measurement time. It should be noted that the data collected from LSDV were not subjected to additional noise removal or any other type of signal pre-processing, and it was not possible to eliminate the noise from the signal completely. The problem of high-level noise was mainly related to the form of low-frequency vibrations, which had a high amplitude. This can be particularly noticeable in Figure 11a,c.

\subsubsection{Damage Localization—Results}

The numerical calculations for the results of the experimental measurements were carried out according to the same research programme as for the simulation stage. First of all, the fit factor for the particular vibration forms was analyzed, and the calculation results are presented in Figure 11. 

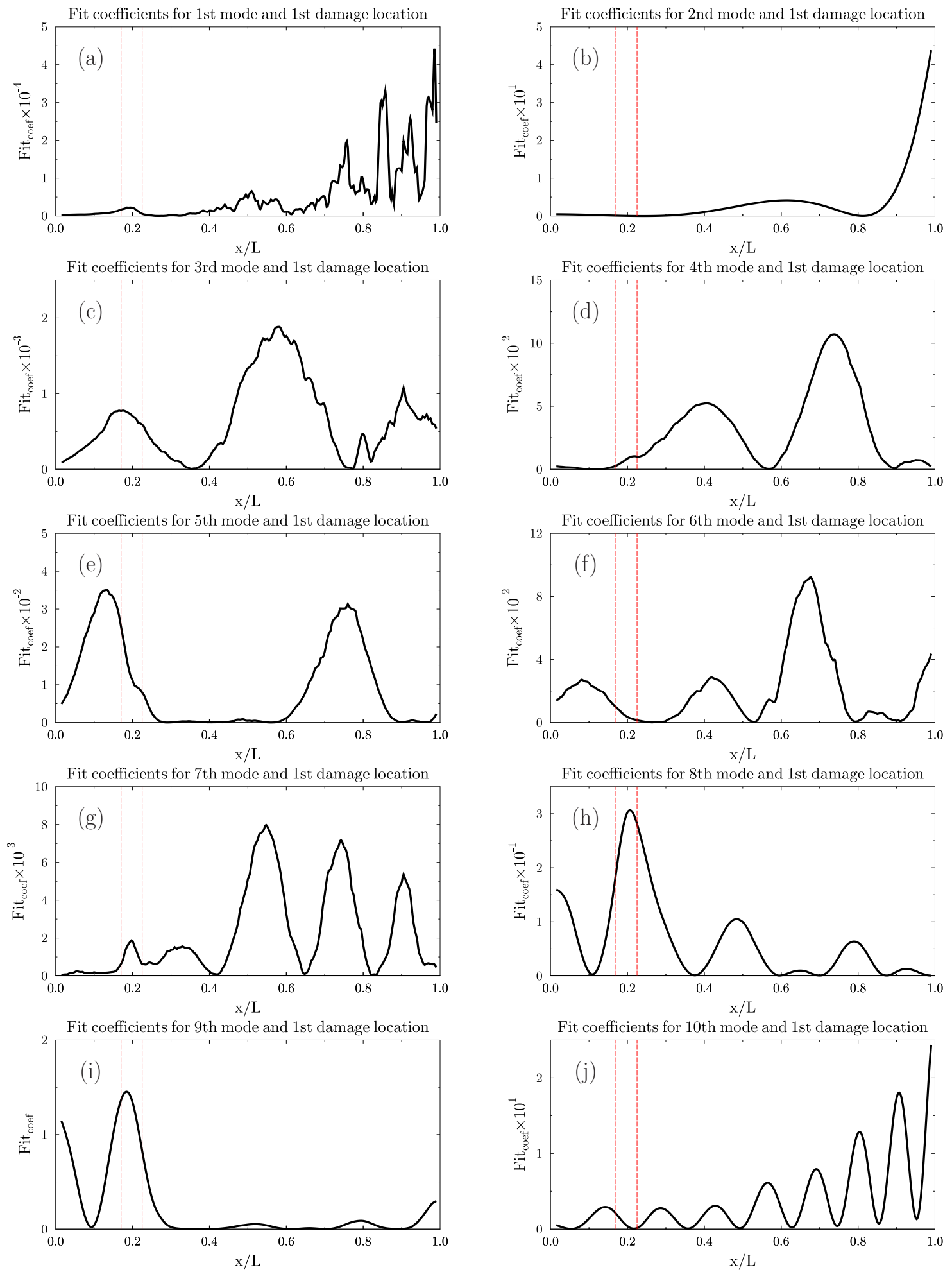

Figure 11. Results of fit coefficient calculation for modes order from 1 to 10 and the first case of damage localization: (a-j) consecutive modes; vertical dashed line-location of the damage; $\mathrm{x} / \mathrm{L}$-normalized position.

In the following order, the fit factor was determined for all available vibration forms averaged by the RMS method for three damage locations. In Figure 12 the curves of the fit factor are shown. 

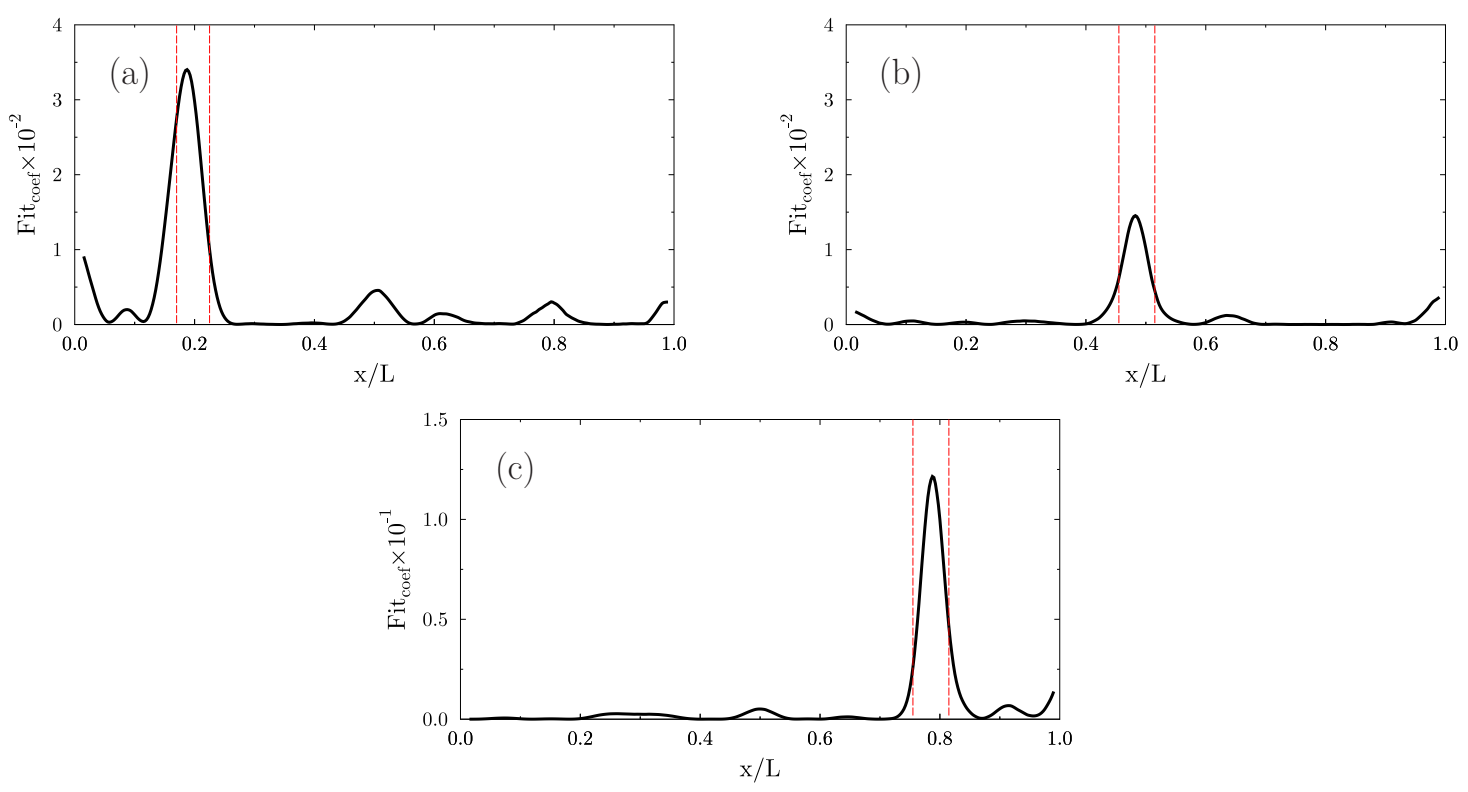

Figure 12. Results of fit coefficient calculation for averaged modes for all cases of damage localization: (a) 1st location; (b) 2nd location; (c) 3rd location; dashed line-location of the damage; $\mathrm{x} / \mathrm{L}$-normalized position.

Finally, the fit factor was determined for the three damage locations near the centre of the blade midspan, as shown in Figure 13.

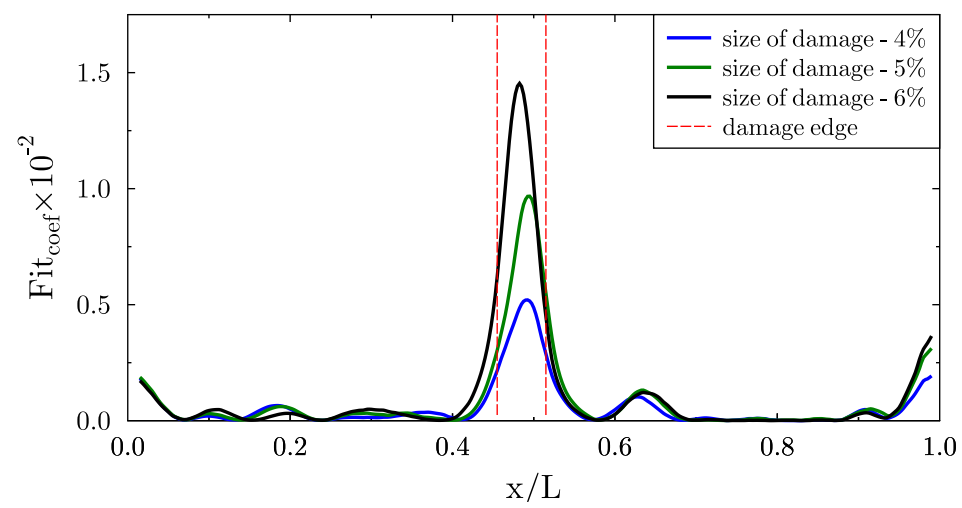

Figure 13. Results of fit coefficient calculation for RMS value for all given modes shapes and three size of damage in one localization; $\mathrm{x} / \mathrm{L}-$ normalized position.

All conclusions that were formulated after the simulation stage were confirmed on experimental data. In the case of single modes analysis, the determination of the damage position was difficult, and only a few vibration forms clearly indicated the damage edges. After the application of averaging by RMS method of all available mode shapes, results were obtained that clearly showed the localization of damage. However, some differences could be seen in relation to the results of the calculation with the use of numerical simulation data. The reasons for these differences should be seen both in the too idealized numerical model, physical limitations of measuring instruments and assumed damage model in the experiment. Even though optimal laboratory conditions were ensured during the tests, the obtained measurement data were noisy. Moreover, the damage model adopted in the experiment (a steel element fixed to the blade surface) was supposed to change the stiffness of the coating locally. However, its additional mass caused a discrepancy in the vibration amplitude for the damaged and reference state, which affected the final calculation results. 


\section{Conclusions}

The present paper introduces a delamination detection and localization technique, which was developed based on the numerical simulations results as well as an experimental test conducted in laboratory conditions. The research was carried out focusing on a specific object which is a composite wind turbine blade. The principle of the presented diagnostic method is to statistically examine changes in the first ten bending mode shapes of the research object regarding the localization of damage. The modal parameters in the meaning of the natural vibrations shapes of the tested blade were assessed calculating the fit coefficients. The calculation procedure was used to search local changes in the combination of the forms of the vibrations that could indicate the presence of delamination. The studies were conducted for several variants of damage location. A total of three damage levels in five positions of the blade coating for simulation calculation were taken into account. For experimental validation of the proposed detection method, data from vibration measurements were collected in three different locations. Additionally, for one location scenario, the measurements for three different sizes of damage area were conducted. For both parts of the presented research, the intact state of the blade was also registered and used in the diagnostic process as a reference signal. In the experimental part of studies, the authors used the high-quality equipment dedicated to non-contact, fast and high accuracy of the vibration measurements. The use of Scanning Laser Vibrometry allowed for optimal accuracy of the collected data with a low level of the noise. Furthermore, the laboratory conditions for the testing procedure enabled to identify the capabilities and limitations resulting from the nature of those type of measurement, which would involve the practical application of the proposed method.

The major advantages of the method are, respectively, the speed of calculation, no signal pre-processing required, as well as only one initial parameter affecting the final analysis effect, i.e., the size of the sliding window. The main disadvantage of this method is the need to know the condition of an intact blade or its predecessor.

One of the main requirements that should be considered when attempting to implement the proposed diagnostic system in practice are the low levels of signal distortion. The adequately good signal-to-noise ratio of measurement data ensures a high level of fault indication while increasing the sensitivity of the method (it is possible to detect smaller faults). Nevertheless, based on the results described in the article, it can be concluded that the diagnostic method based on the analysis of the shapes of natural vibrations in a complex form using statistical tools is effective even with relatively small damage. Moreover, the use of a combination of the presented diagnostic method and a sensor system built into the blade of the wind turbine would allow for the condition monitoring of the object in real-time. Multiple measurements would enable reducing the influence of measurement noise or accidental errors in data on the detection result, and historical measurements could be used as a reference state. Combining the presented method with other more advanced signal processing techniques (e.g., wavelet analysis or AI techniques) will allow not only to determine the exact edge of the defect but perhaps also to predict the speed and direction of defect formation.

The further studies related to the method presented in the paper, aimed at both improving it and identifying possible limitations in its practical application, will be concentrated on:

- analyzing of the torsional vibration mode shapes of the blade for the statistical calculation,

- testing the influence of measuring noise level on the effectiveness of damage detection and localization,

- the calculation for 2-dimensional forms of vibration,

- increasing the sensitivity of damage edge detecting by combining the statistical method with wavelet analysis,

- testing the effectiveness of the method on data from different measuring techniques.

Author Contributions: The data analysis and interpretation was performed by L.D. under the super vision of M.K.; the manuscript was drafted by L.D., reviewed by M.K. All authors have read and agreed to the published version of the manuscript. 
Funding: This research received no external funding.

Acknowledgments: Calculations were carried out at the Academic Computer Centre in Gdansk.

Conflicts of Interest: The authors declare no conflict of interest.

\section{References}

1. Meng, H.; Lien, F.S.; Glinka, G.; Geiger, P. Study on fatigue life of bend-twist coupling wind turbine blade based on anisotropic beam model and stress-based fatigue analysis method. Compos. Struct. 2019, 208, 678-701. [CrossRef]

2. Jang, Y.J.; Choi, C.W.; Lee, J.H.; Kang, K.W. Development of fatigue life prediction method and effect of 10-minute mean wind speed distribution on fatigue life of small wind turbine composite blade. Renew. Energy 2015, 79, 187-198. [CrossRef]

3. Nijssen, R.P.L.; Brøndsted, P. 6-Fatigue as a design driver for composite wind turbine blades. In Woodhead Publishing Series in Energy, Advances in Wind Turbine Blade Design and Materials; Brøndsted, P., Nijssen, R.P.L., Eds.; Woodhead Publishing: Cambridge, UK, 2013; pp. 175-209.

4. Yang, B.; Sun, D. Testing, inspecting and monitoring technologies for wind turbine blades: A survey. Renew. Sustain. Energy Rev. 2013, 22, 515-526. [CrossRef]

5. Beganovic, N.; Soffker, D. Structural health management utilization for lifetime prognosis and advanced control strategy deployment of wind turbines: An overview and outlook concerning actual methods, tools, and obtained results. Renew. Sustain. Energy Rev. 2016, 64, 68-83. [CrossRef]

6. Du, Y.; Zhou, S.; Jing, X.; Peng, Y.; Wu, H.; Kwok, N. Damage detection techniques for wind turbine blades: A review. Mech. Syst. Signal Process. 2020, 141, 106445. [CrossRef]

7. Sarrafi, A.; Mao, Z.; Niezrecki, C.; Poozesh, P. Vibration-based damage detection in wind turbine blades using Phase-based Motion Estimation and motion magnification. J. Sound Vib. 2018, 421, 300-318. [CrossRef]

8. Chen, B.; Yu, S.; Yu, Y.; Zhou, Y. Acoustical damage detection of wind turbine blade using the improved incremental support vector data description. Renew. Energy 2020, 156, 548-557. [CrossRef]

9. Janeliukstis, R.; Rucevskis, S.; Wesolowski, M.; Chat, A. Experimental structural damage localization in beam structure using spatial continuous wavelet transform and mode shape curvature methods. Measurement 2017, 102, 253-270. [CrossRef]

10. He, M.; Yang, T.; Du, Y. Nondestructive identification of composite beams damage based on the curvature mode difference. Compos. Struct. 2017, 176, 178-186. [CrossRef]

11. Wu, R.; Zhang, D.; Yu, Q.; Jiang, Y.; Arola, D. Health monitoring of wind turbine blades in operation using three-dimensional digital image correlation. Mech. Syst. Signal Process. 2019, 130, 470-483. [CrossRef]

12. Sanati, H.; Wood, D.; Sun, Q. Condition Monitoring of Wind Turbine Blades Using Active and Passive Thermography. Appl. Sci. 2018, 8, 2004. [CrossRef]

13. Shahsavari, V.; Chouinard, L.; Bastien, J. Wavelet-based analysis of mode shapes for statistical detection and localization of damage in beams using likelihood ratio test. Eng. Struct. 2017, 132, 494-507. [CrossRef]

14. Ulriksen, M.D.; Damkilde, L. Structural damage localization by outlier analysis of signal-processed mode shapes-Analytical and experimental validation. Mech. Syst. Signal Process. 2016, 68-69, 1-14. [CrossRef]

15. Eder, M.A.; Belloni, F.; Tesauro, A.; Hanis, T. A multi-frequency fatigue testing method for wind turbine rotor blades. J. Sound Vib. 2017, 388, 123-140. [CrossRef]

16. Reddy, A.; Indragandhi, V.; Ravi, L.; Subramaniyaswamy, V. Detection of Cracks and damage in wind turbine blades using artificial intelligence-based image analytics. Measurement 2019, 147, 106823. [CrossRef]

17. Ghiasi, R.; Ghasemi, M.R.; Noori, M. Comparative studies of metamodeling and AI-Based techniques in damage detection of structures. Adv. Eng. Softw. 2018, 125, 101-112. [CrossRef]

18. Yang, K.; Rongong, J.A.; Worden, K. Damage detection in a laboratory wind turbine blade using techniques of ultrasonic NDT and SHM. Strain 2018, 54, e12290. [CrossRef]

19. Doliński, Ł.; Krawczuk, M.; Żak, A. Detection of Delamination in Laminate Wind Turbine Blades Using One-Dimensional Wavelet Analysis of Modal Responses. Shock Vib. 2018, 2, 1-15. [CrossRef]

20. Doliński, Ł.; Krawczuk, M.; Żak, A. Damage Detection in the Wind Turbine Blade Using Root Mean Square and Experimental Modal Parameters. In Lecture Notes in Mechanical Engineering, Proceedings of the 13th International Conference on Damage Assessment of Structures, Porto, Portugal, 9-10 July 2019; Wahab, M., Ed.; Springer: Singapore, 2020; pp. 728-742. 
21. Tang, J.; Soua, S.; Mares, C.; Gan, T.H. A Pattern Recognition Approach to Acoustic Emission Data Originating from Fatigue of Wind Turbine Blades. Sensors 2017, 17, 2507. [CrossRef]

22. Hoell, S.; Omenzetter, P. Fukunaga-Koontz feature transformation for statistical structural damage detection and hierarchical neuro-fuzzy damage localisation. J. Sound Vib. 2017, 400, 329-353. [CrossRef]

23. Colone, L.; Hovgaard, M.K.; Glavind, L.; Brincker, R. Mass detection, localization and estimation for wind turbine blades based on statistical pattern recognition. Mech. Syst. Signal Process. 2018, 107, $266-277$. [CrossRef]

24. Ansys, Vibration Simulation, Measurement and Analysis. Available online: https://www.ansys.com/ products/structures/vibrations. (accessed on 24 August 2020)

25. MATLAB Documentation, Available online: https://www.mathworks.com/help/matlab/ (accessed on 24 August 2020)

26. Bagherpoor, T.; Li, X. Structural optimization design of $2 \mathrm{MW}$ composite wind turbine blade. Energy Procedia 2017, 105, 1226-1233. [CrossRef]

27. Vibrometry-Polytec. Available online: https://www.polytec.com/eu/vibrometry/ (accessed on 24 August 2020).

(C) 2020 by the authors. Licensee MDPI, Basel, Switzerland. This article is an open access article distributed under the terms and conditions of the Creative Commons Attribution (CC BY) license (http:/ / creativecommons.org/licenses/by/4.0/). 